\title{
Moyen-Orient : l'incubation démocratique malgré tout
}

\author{
Pierre Blanc \\ Rédacteur en chef de Confluences Méditerranée, enseignant-chercheur en géopolitique à Sciences \\ Po Bordeaux (Les Afriques dans le monde) et Bordeaux Sciences Agro.
}

\section{Haoues Seniguer}

Maître de conférences en science politique à Sciences Po Lyon (Laboratoire Triangle, UMR 5206).

S'interroger sur la démocratie au Moyen-Orient est un exercice à tout le moins délicat. Délicat car cette région n'est pas un objet géopolitique clairement sérié et encore moins homogène. S'il est avant tout «le produit d'un imaginaire géopolitique $»^{1}$, nous retiendrons ici le bloc que composent les pays du Levant, autour desquels se trouvent la péninsule arabique au Sud-Est, la Turquie au Nord-Ouest, l'Égypte au Sud-Ouest et l'Iran au Nord-Est. Autant dire des pays hétérogènes par la culture, la langue et l'histoire, mais liés par des dynamiques géopolitiques et économiques.

Délicat également car les situations de guerre civile en Syrie, en Irak et au Yémen rendent quelque peu incongru ce questionnement sur la démocratie à l'heure où la reconstruction semble moins que jamais à l'ordre du jour tandis que les États s'y effondrent. Mais par-delà ces tragédies, l'exercice est difficile car il est peu loisible d'embrasser la diversité des situations politiques au vu de la densité de cette aire. Par ailleurs, comment apprécier pleinement la nature démocratique de tous ces régimes en quelques mots, sinon à postuler illusoirement l'existence d'une démocratie chimiquement pure, à savoir une réalité sociopolitique qui correspondrait idéalement, trait pour trait, aux critères théoriques élaborés par nombre de penseurs de la démocratie ? Puis, quel type de démocratie peut-il à ce point être érigé en modèle que celui-ci en deviendrait acceptable partout et pour tous ? Ce débat, quoique important et crucial en philosophie politique, n'est pas à proprement parler du ressort du sociologue du politique ou des relations internationales. Nos ambitions seront donc plus modestes et essentiellement tournées vers ou sur l'existant.

1 Hamit Bozarslan, Sociologie politique du Moyen-Orient, Paris, La Découverte, 2011, p. 7. 


\section{Autoritarisme dominant, démocratie malade}

Par-delà sa diversité politique, la carte du Moyen-Orient fait apparaître une nette prévalence de l'autoritarisme, à des degrés divers, soit sous forme monarchique (pays du Golfe et Jordanie), soit sous forme républicaine (Égypte et Iran). Ici comme ailleurs, on voit que la forme du régime ne prédispose ni n'indispose en principe à l'autoritarisme ou à la démocratie ${ }^{2}$. Dans ce premier coup d'œil, on exclut ici les situations de guerre civile (Syrie, Irak et Yémen) : les catégories autoritarisme / démocratie paraissent inopérantes du fait que ces guerres sont en grande partie l'aboutissement de logiques autoritaires et prédatrices antérieures ${ }^{3}$. À l'échelle mondiale, le Moyen-Orient - associé au Maghreb - est la région qui apparaît la plus autoritaire selon l'indice de démocratie calculé chaque année par l'Economist Group. En 2016, il y était en effet de 3,56, contre environ 8,5 en Europe de l'Ouest et en Amérique du Nord, et 4,37 pour l'Afrique hors Afrique du Nord.

Dans ce paysage régional, la démocratie s'est donc pour l'heure peu fixée, et encore, le cas échéant, elle revêt une forme très particulière ou semble mise à mal : la démocratie israélienne est certes la plus aboutie dans la région, et ce comparativement, mais sa politique coloniale, qui ne cesse de s'approfondir dans les Territoires palestiniens occupés, en vient à saper les fondations sur lesquelles elle prétend être assise. La confirmation au pouvoir de Benyamin Netanyahou, en 2015, laisse toujours plus ouvert le boulevard à une extrême droite messianiste qui s'impose au centre du jeu et qui ne veut pas de paix avec les Palestiniens, et encore moins de deux États dotés de frontières sûres ${ }^{4}$. La dépossession territoriale de la Cisjordanie et le blocus contre Gaza consacrent tous les jours le «politicide», selon l'expression de Baruch Kimmerling, qui consiste à entraver la construction d'un toit politique pour les Palestiniens. Faut-il aussi rappeler que le pouvoir en place n'a de cesse de mettre sous contrôle les organisations de plaidoyer israéliennes (B'Tselem, Gush Shalom, Breaking the Silence, etc.) qui veulent en finir avec le colonialisme de leur pays, en ayant notamment adopté en juillet 2016 une loi qui les oblige à la transparence des financements ?

2 Voir le cas des monarchies modernes, espagnole ou britannique notamment.

3 Voir Pierre Blanc et Jean-Paul Chagnollaud, L'invention tragique du Moyen-Orient, Paris, Autrement, 2017.

4 Force est d'admettre que cette brutalité n'est plus toujours perçue dans une France confrontée, depuis 2012, à une série d'attentats et d'assassinats au nom de l'islam, et où l'on a vu des personnalités françaises, intellectuels, journalistes ou politiques, vanter le savoir-faire israélien en matière sécuritaire, allant jusqu'à demander qu'on le copie. 
Quant à la démocratie libanaise, fondée sur le consociativisme, elle n'est pas encore sortie du communautarisme, puisque les individus, et plus encore les politiques, ne peuvent échapper à l'assignation communautaire. Les « fromagistes » ainsi nommés par le président Fouad Chehab (19581964), autrement dit les chefs communautaires, prennent encore ce pays comme une ressource politique et économique à se partager, qui est l'expression manifeste d'une gestion patrimonialiste. Enfin, pour ce qui est de la Turquie, on perçoit la prégnance de plus en plus aigüe d'un pouvoir personnel du président Erdogan, qui fait preuve, malgré la démocratie électorale, d'autoritarisme à l'égard d'opposants réprimés et emprisonnés. Sans être la seule cible, le parti parlementaire kurdiste pâtit énormément de ce redéploiement autoritaire. Le Parti démocratique des peuples (HDP), qui dispose d'une bonne base électorale, a en effet vu certains de ses élus, notamment des maires, écroués sur simples soupçons. Les partisans du «non »au référendum du 16 avril 2017, qui a débouché sur le renforcement des pouvoirs de la présidence, ont été fortement stigmatisés et parfois assimilés à des «terroristes » et aux adeptes du coup d'État avorté du 15 juillet 2016.

Face à cette large prédominance du fait autoritaire, la démocratie a été au cœur des réflexions sur sa résilience dans la région. En fait, cette question s'est surtout posée dans le cadre du dit monde arabe, avant que le mouvement de révoltes en 2011 ne vienne remettre en question cette représentation largement fantasmée ou mythifiée d'une démocratie / démocratisation impossible. S'il est possible, avec le politiste Michel Camau, de parler de "phénomène d'émulation protestataire à une échelle arabe, suite aux révoltes de 2011-2012, en dépit des différences de contexte et de structure $»^{5}$, ce dernier ne manque pas de préciser dans le même temps à la fois leur caractère inédit, en particulier par «leur double dimension nationale et internationale », et l'impossibilité de postuler un «modèle invariant des révolutions ». Ce qu'il est en revanche permis d'affirmer assurément, c'est une sorte de mise en cause majeure, sans précédent, de l'assignation autoritaire qui a profité aux élites politiques des régimes concernés, ou à nombre de pouvoirs extérieurs, qui ont vu dans cet autoritarisme un gage de stabilité et d'endiguement des migrations et de confinement du terrorisme.

5 Michel Camau, «Un printemps arabe ? L'émulation protestataire et ses limites », L'Année du Maghreb 2012, VIII, 2012, p. 27. 


\section{La remise en question de l'assignation autoritaire}

À l'heure où la démocratie semblait se généraliser dans le sillage de l'effondrement du bloc soviétique à la fin des années 1980, au point que le chercheur Francis Fukuyama annonçait même triomphalement la fin de l'Histoire par la victoire annoncée du libéralisme politique à l'échelle mondiale, le Moyen-Orient et plus largement le monde arabe ont été regardés avec plus d'insistance, sinon de condescendance, comme des «exceptions autoritaires », selon l'expression de Michel Camau, qui lui-même ne souscrivait pas à ce regard réducteur, pas plus

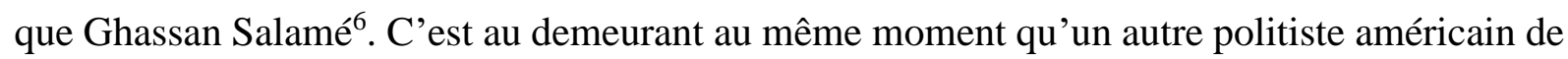
renom, celui qui, avec Bernard Lewis, prophétisera le choc des civilisations, Samuel Huntington, rédigea un article sur «la troisième vague démocratique $»^{7}$. Son propos est un parfait condensé de vues historicistes et culturalistes. En somme, croyant fermement en l'existence de cultures fixes ou figées, les obstacles à la démocratisation, dont les pays arabes majoritairement musulmans sont l'archétype, seraient au nombre de trois : culturel, économique et politique. Dans ce genre d'analyse, l'élément culturel et / ou religieux tient lieu de premier référent analytique. En particulier, l'islam étant vu comme la religion de la soumission, il préparerait l'homo islamicus à accepter toute forme d'allégeance politique, à commencer par la dictature, ou à la favoriser. Autrement dit, la matrice islamique se présenterait comme un obstacle démocratique permanent ${ }^{8}$.

À supposer que ce raccourci «islam = autoritarisme » ait quelque pertinence par certains aspects, particulièrement par les accents liberticides et autoritaristes de l'islamisme - qui est une lecture essentialiste de l'islam - qui exploite à plein la fibre identitaire de l'islam, faut-il réduire tout État, société, homme ou femme à une seule influence, à une seule dimension ou encore à une seule identité ? Maxime Rodinson avait ainsi nommé «théologocentrisme » cette attitude consistant à affirmer le primat de l'islam dans la conduite de ceux qui s'en réclament. C'est, au demeurant, ce réflexe culturaliste qui pave de nos jours abondamment la voie au racisme antimusulman ou à l'islamophobie. Refusant donc cette idée que les choix personnels sont

6 Lire Ghassan Salamé, «Sur la causalité d'un manque : pourquoi le monde arabe n'est-il donc pas démocratique ?», Revue française de science politique, vol. 41, $\mathrm{n}^{\circ} 3,1991$.

7 Samuel Huntington, « Democracy Third Wave », Journal of Democracy, vol. 2, n 2, printemps 1991.

8 De façon moins savante, l'écrivain français Alain Finkielkraut a d'ailleurs fait montre, dès le début des révoltes populaires, d'un fort scepticisme en convoquant le précédent iranien, pour prédire que le pire est - toujours - à venir, en raison de la ligne d'horizon islamiste qui se dessinait, même si les mots d'ordre des manifestants étaient avant tout d'ordre séculier : liberté, dignité, fin de la corruption et de la dictature, etc. 
uniquement sous l'emprise d'une stricte loi religieuse, l'islamologue s'exprimait encore ainsi : «L'essentiel est que je me refuse à considérer l'islam comme une totalité intellectuelle, un système d'idées, de pratiques, de choix de vie qui seraient à la racine ou le noyau de tous les comportements publics et privés du monde qui fait profession d'adhérer à cette religion. » ${ }^{9}$ L'argument anthropologique basé sur la prédominance du patriarcat ${ }^{10}$ occupait également une bonne place dans un regard orientaliste ou néo-orientaliste aux préjugés négatifs marqués, le rapport très vertical à l'autorité du père étant supposé se projeter dans l'espace public arabe, dans lequel la figure du chef n'était jamais que le « sur-père » - l'espace public étant appréhendé comme le prolongement de l'espace domestique ou familial, avec toutes les dérives que cela suppose. Sur le plan économique, le profil globalement rentier servait aussi d'explication. D'une part, parce que les États riches de leur sous-sol dans la région n'avaient pas à se soumettre au principe de «redevabilité » (accountability), étant donné qu'ils n'avaient pas besoin de prélever l'impôt. D'autre part, leur richesse pouvait leur permettre un investissement lourd dans l'appareil autoritaire ${ }^{11}$ et dans l'achat de la paix sociale.

Cette courte évocation n'épuise pas l'étendue des causes évoquées pour expliquer la survivance autoritaire dans la région, qui tient à des paramètres multiples. Il ne s'agit pas pour autant d'en faire ici une critique systématique, surtout depuis que les mobilisations en Iran en 2009 et dans les pays arabes à partir de 2011 sont venues en montrer la limite, voire, pour certaines, l'inanité. L'Iran, un pays corseté depuis des décennies, d'abord sous la poigne du chah (1941-1979), puis sous la férule des partisans du wilayat al-faqih (gouvernement du jurisconsulte) à partir de 1979, a révélé que l'«autoritarisme électoral » n'était pas sans fragilité. Le résultat des élections de juin 2009, qui donnait la victoire à Mahmoud Ahmadinejad au détriment de Mir Hossein Moussavi, fut en effet fortement contesté par une opposition persuadée que les urnes avaient surtout révélé une fraude d'envergure. Il s'agissait d'une expression forte de la conscience démocratique dans un paysage politique officiel autoritaire. Les marges démocratiques existent donc bel et bien.

Dans le reste du Moyen-Orient mais aussi dans tout le monde arabe, où quelques mouvements sociaux et politiques antérieurs peuvent apparaître a posteriori comme les prodromes d'un soulèvement plus général, c'est bien une vague révolutionnaire, partie de Sidi Bouzid, qui avait

\footnotetext{
${ }^{9}$ Maxime Rodinson, L'Islam : politique et croyance, Paris, Fayard, 1993, p. 9.

10 Hisham Sharabi (dir), Neopatriarchy. A Theory of Distorted change in Arab Society, New York, Oxford University Press, 1988.

${ }^{11}$ Luis Martinez, Violence de la rente pétrolière. Algérie, Irak, Libye, Paris, Presses de Sciences Po, 2009.
} 
spectaculairement fini par emporter plusieurs pouvoirs et fragilisé d'autres. « Réjouissons-nous des révoltes arabes », écrivait Faouzia Zouari ${ }^{12}$, comme en écho aux Considérations sur le malheur arabe de Samir Kassir, assassiné en 2005. C'était en mars 2011, alors que son pays la Tunisie - venait de se libérer de son despote sans l'appui de F-16... Elle voyait aussi le fait que la peur avait changé de camp, que les femmes s'étaient mobilisées, que le népotisme et la corruption, mais aussi l'arrogance et la brutalité des despotes s'annonçaient bientôt renvoyées aux oubliettes de l'Histoire. Plus encore, le mouvement semblait en finir avec cette vieille idée tenace qui voulait que les sociétés arabes étaient vouées à accepter culturellement et religieusement la chape de plomb de l'autoritarisme, qu'il soit religieux ou politique, voire les deux à la fois.

\section{L’idée démocratique : entre extinction, écrasement et résilience}

Ces révoltes ont connu des fortunes diverses, mais force est d'admettre que le répertoire de la coercition est large, depuis les apparentes concessions au traitement militaire massif. Dans les pays du Golfe, le désamorçage s'est opéré par l'achat de la paix sociale que facilite la rente (Arabie saoudite et Koweït) et par la réforme politique, notamment en Jordanie où quelques changements cosmétiques ont été opérés. Ailleurs, comme en Égypte, on note un redéploiement autoritaire répressif depuis le coup d'État militaire du 3 juillet 2013, l'arrivée au pouvoir du maréchal Abdel Fattah al-Sissi et la répression systématique des opposants, Frères musulmans en tête, avant que les organisations indépendantes des droits de l'homme soient à leur tour éliminées. Ceci montre, s'il fallait en douter, que la répression de la confrérie n'est in fine qu'un prétexte pour venir à bout de toute opposition politique, fût-elle la plus à la pointe de demandes démocratiques. Au Yémen, à feu et à sang depuis 2014, la révolte des Houthis a entraîné une réaction brutale du régime de Sanaa, épaulé par une coalition armée menée par l'Arabie saoudite. Au Bahreïn, les mobilisations ont été fortement entravées par l'intervention militaire des pays du Conseil de coopération du Golfe (CCG) effrayés par le risque d'une propagation de la contestation sur leurs territoires, alors même que le Qatar se présentait ailleurs dans le monde arabe comme le soutien indéfectible des aspirations démocratiques populaires. En Syrie, depuis 2011, c'est par la brutalisation la plus extrême que le régime, avec ses alliés russe, iranien et les milices chiites d'Irak et du Liban, pilonne sans discontinuer opposants et civils, forçant

12 Faouzia Zouari, « Réjouissons-nous des révoltes arabes », Confluences Méditerranée, n 77, L'Harmattan, printemps 2011. 
des millions de personnes à l'exil. De son côté, l'opposition a vu ses secteurs les plus violents se renforcer, alors que ceux qui étaient les plus disposés à un compromis ont été marginalisés, pour ne pas dire éliminés. Quant au mouvement vert en Iran, il a été écrasé par les gardiens de la révolution associés à leurs supplétifs, les bassidji de la République islamique, très présents dans l'appareil sécuritaire du pays. On pourrait enfin ajouter que le fédéralisme irakien institué par la Constitution de 2005 n'a pas empêché la mainmise des clans proches du nouveau pouvoir sur les ressources de l'État central à Bagdad, tandis que dans la région fédérée du Gouvernorat kurde du Nord, on assiste à une sorte de prédation assez autoritaire de la part du Parti démocratique du Kurdistan ${ }^{13}$. Il en est de même dans la république autoproclamée du Rojava en Syrie, où le Parti de l'union démocratique (PYD), lié au Parti des travailleurs du Kurdistan (PKK) turc, assure un leadership brutal.

Ce tableau apparemment sombre ne doit toutefois pas occulter la permanence ou la rémanence d'un socle démocratique, plus ou moins organisé et incandescent suivant les cas, dans chacun des pays en question. Dans son suivi des opinions publiques, l'Arab Center for Research and Policy Studies montre la résilience du désir de démocratie dans la région, en dépit des vicissitudes qu'elle subit. En 2016, 73 \% des personnes interrogées d'un large échantillon voyaient la démocratie comme le meilleur système politique. Certes, dans cette enquête, il faut noter quand même un certain recul de l'espérance politique puisqu'en 2016, $45 \%$ des personnes interrogées contre $60 \%$ en 2014 déclaraient que « les printemps arabes affrontent des obstacles mais qu'ils réaliseront leur buts ».

Même dans le cas de la Syrie, où la clôture autoritaire s'est muée en bombes létales contre des civils, tandis qu'une opposition armée hétéroclite, composée entre autres d'islamistes radicaux, commet aussi des crimes de guerre, nous ne saurions à notre tour oublier les acteurs sociaux, individuels ou collectifs, mobilisés depuis 2011 pour la fin de la dictature, l'arrêt des violences de part et d'autre, et l'avènement «d'une Syrie démocratique et d'un État civil ». Dès 2012, en effet, la Syrie a vu fleurir dans les villes et villages éloignés de l'emprise du pouvoir loyaliste des comités chargés de faire tout ce que l'État ne faisait plus. À l'origine de ces conseils locaux, on trouve des militants issus des «coordinations» (tansiqiyyat) des premiers temps du soulèvement syrien.

13 Voir Adel Bakawan, «L'échec du nationalisme kurde : fragmentation, partisanisation, milicisation », Confluences Méditerranée, ${ }^{\circ}$ 100, iReMMO - L'Harmattan, printemps 2017 ; et Arthur Quesnay et Yohanan Benhaim, «L'espace politique kurde dans le conflit syrien : intégration régionale et polarisation partisane », Confluences Méditerranée, nº 98, iReMMO - L'Harmattan, automne 2016. 
Un acte important, mais vite oublié et même marginalisé - compte tenu des rapports de forces amplement défavorables sur le terrain -, a été réalisé dans le cadre de la conférence internationale de Genève (28-29 janvier 2013), principalement sous l'égide de l'Institut scandinave pour les droits humains et de la Commission arabe pour les droits de l'homme ${ }^{14}$. Les discours ou déclarations qui y ont été tenus méritent d'être mis en évidence tant les perturbations et l'arsenal répressif déployé sans distinction aucune sur les populations civiles sont meurtriers, et invisibilisent ce faisant les revendications de celles et ceux qui ont fait un autre choix que celui du régime ou de l'opposition islamiste armée. Parmi les participants, dont des religieux, tous convergeaient pour réaffirmer la nécessité de la chute du régime de Bachar Al-Assad, reconnu pour son caractère hautement meurtrier, de l'arrêt total de l'escalade militaire et le refus de toute ingérence étrangère, pro ou anti-régime. On peut, en guise d'illustration, souligner les mots importants prononcés par l'un des initiateurs principaux du mouvement, Haytham Manâ', à l'adresse des partisans de la révolte syrienne : «La religion est à Allah et la nation à tous »; « la Syrie est à tous ses enfants »; « ne tue pas ; ne kidnappe pas ; ne vole pas ; ne sois pas injuste ; ne sois pas affecté ; ne corromps pas ; n'excommunie pas ; ne trahis pas ; n'ostracise pas ; ne détruis pas $»^{15}$, etc. La présence du cheikh syrien Riyâd Drâr à ce sommet est également à relever : il est l'infatigable partisan d'un islam laïc ou compatible avec la laïcité, d'une démocratie syrienne «qui évite que le sang ne coule », «qui mette un terme à toute forme de violence » et «à l'extrémisme religieux », par «la tenue d'élections équitables et transparentes $»^{16}$. Ces perspectives paraissent lointaines et improbables, étant donné la répression intense que déploient le régime Assad et ses alliés, ainsi que l'apathie de la diplomatie internationale.

\section{Des incubateurs à observer}

Malgré sa brutalité, la tragédie syrienne, pas davantage que ce qui a cours au Yémen et en Irak, n'autorise donc à faire l'éloge funèbre des soulèvements initiés dans la région, tant les processus d'affirmation démocratique sont longs ${ }^{17}$, diversifiés, contradictoires et parfois violents. Il y a

14 « Le Congrès syrien international pour une Syrie démocratique et un État civil », Genève, 28-29 janvier 2013, organisé par l'Institut scandinave pour les droits humains, Éditions Ashtarowt, 2013 (en arabe).

15 Ibid., pp. 9-10.

16 Ibid., pp. 28-34.

17 Cent vingt-cinq ans après la Révolution française, le Chinois Zhou Enlai avançait l'idée qu'il était bien trop prématuré d'en analyser les conséquences. 
certes un sentiment de grande confusion, d'incrédulité démocratique si l'on veut, face à l'actualité immédiate du Moyen-Orient. Mais c'est parce que beaucoup d'observateurs eurent une lecture téléologique des événements révolutionnaires au Moyen- Orient, mais aussi au Maghreb, entre 2010 et 2012 essentiellement, qu'une grande confusion s'ensuivit. Une mauvaise appréciation initiale a, ce faisant, conduit à des mésinterprétations ou à des contresens majeurs sur l'appréciation des dynamiques sociopolitiques à l'œuvre. Pire, elle mène ou mena à un certain désenchantement. Pourtant, la demande de dignité, la fin de la corruption et le desserrement de l'étreinte politique ${ }^{18}$ n'ont effectivement pas déserté une arène qui n'est sans doute pas revenue au statu quo ante, en dépit des reprises en main autoritaires, depuis la brutalité politique redoublée au désamorçage électoral vaguement réformateur.

Au regard du canon démocratique, les pays de la région sont certes loin de réunir l'entièreté des critères. Remarquons à ce sujet que les parangons de la démocratie que sont les États-Unis ou la vieille Europe traversent eux-mêmes une sorte de «déprise démocratique », qui préfigure une certaine faillite ${ }^{19}$ de modèles qu'ils prétendaient incarner et diffuser à l'extérieur. Raffaele Simone, l'auteur de ce diagnostic, retient quantité de critères, dont la plupart des pays dits démocratiques s'éloignent. Il envisage aussi trois piliers de la démocratie - les institutions démocratiques, la mentalité démocratique et la mythologie démocratique - pour constater qu'ils vacillent aussi, et pour souligner en outre que l'avenir semble condamné à une « démocratie faible $»^{20}$.

Dans le cas du Moyen-Orient, il serait vain d'appliquer pour l'heure une même grille, justement parce que la démocratie est encore loin d'y avoir élu domicile. Pour autant, si l'on ne doit pas se garder d'observer les institutions politiques - séparation des pouvoirs, processus électoraux, représentativité - pour constater que pour l'heure, le paradigme démocratique est encore loin d'y être opérant, il convient aussi de prendre davantage en considération les incubateurs qui préparent une transformation du rapport au politique dans le sens des avancées de la liberté : le rejet de la corruption par l'opinion publique, le degré de diffusion de l'éducation - notamment l'enseignement supérieur -, le développement inclusif, l'insertion sociale, économique et politique des femmes, la vigueur de la société civile, la dé-segmentation communautaire,

\footnotetext{
18 Dans les enquêtes de l'Arab Center for Research and Policy Studies, les rejets de la corruption et de l'autoritarisme sont mentionnés en premier dans les raisons des révoltes arabes. Les conditions économiques viennent en troisième. « Findings from the 2016 Arab Opinion Index announced », mars 2017.

${ }^{19}$ Raffaele Simone, Si la démocratie fait faillite, Gallimard, coll. « Le débat », 2015.

20 «Findings from the 2016 Arab Opinion Index announced », op. cit.
} 
l'autonomie culturelle, la liberté de la presse, la circulation des biens et des personnes ou bien la sécularisation sont autant d'éléments sans lesquels la démocratie, même faible, pourrait attendre encore longtemps avant de s'installer au Moyen-Orient. Or les événements révolutionnaires de 2011 ont montré que certains parmi eux sont bien à l'œuvre dans les sociétés de la région, quoiqu'on ne les ait pas regardés suffisamment.

En effet, il a été souligné en amont que les critères classiques de la démocratie, tels qu'ils furent élaborés pour l'essentiel en Europe de l'Ouest ainsi qu'en Amérique du Nord, et qui ont encore largement cours dans ces régions du monde et bien d'autres, s'essoufflent et s'érodent toutefois immanquablement. Une crise de la représentation politique semble aussi consommée que généralisée. Or, justement, dans une partie du Moyen- Orient et du monde arabe en particulier, nous assistons bel et bien, çà et là, à des mobilisations et des expériences sociopolitiques renouvelées, qui paraissent être en mesure d'influencer et de réajuster les politiques gouvernementales, en déplaçant les centres de décision du centre vers les périphéries. La longue parenthèse autoritaire, et parfois la mémoire encore incandescente des violents conflits du passé accroissent la vigilance des sociétaires et des organisations non gouvernementales. En Tunisie, bien que située hors Moyen-Orient, l'on assiste régulièrement à des manifestations ou des plates-formes contre la corruption, pour soutenir l'acheminement vers des formes délibératives de démocratie à travers une dynamisation des pratiques délibératives au niveau des collectivités locales, etc. Il s'agit en quelque sorte de rendre plausible l'accountability, en ce sens qu'il s'agit de préserver la flamme toujours fragile de la démocratie, et qui serait, en l'occurrence, une espèce d'intermédiaire civile entre le mandat impératif et le mandat électoral. N'oublions pas, à cet égard, que les révoltes dans une partie du monde arabe à l'orée de l'année 2011, inspirèrent, d'une manière ou d'une autre, d'autres mouvements protestataires en Europe, comme celui des « indignés » en Espagne.

Il va sans dire que la cristallisation de la demande démocratique ne saurait se faire en l'absence d'apaisement géopolitique : l'atténuation de la rivalité de puissance entre l'Arabie saoudite et l'Iran qui attise la fitna islamique, la fin des guerres civiles en Syrie et au Yémen, l'effondrement du djihadisme et le règlement de la question de la Palestine qui, quoi qu'on en pense, garde une certaine centralité, constituent les éléments de cette facilitation démocratique. 